\title{
Geocronología de los yacimientos achelenses de Pinedo y Cien Fanegas (Valle del Tajo) e implicaciones en la evolución fluvial en el entorno de Toledo (España)
}

\section{Geochronology of the Acheulean sites of Pinedo and Cien Fanegas (Tagus River valley), and implications for the fluvial evolution in the environs of Toledo (Spain)}

\author{
M. López-Recio ${ }^{1}$, P.G. Silva ${ }^{2}$, E. Roquero ${ }^{3}$, P.P. Cunha ${ }^{4}$, F. Tapias ${ }^{5}$, M. Alcaraz-Castaño ${ }^{6}$,

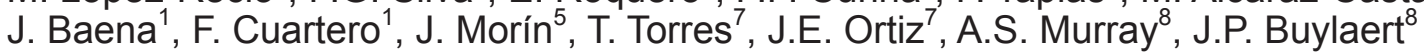 \\ 1 Departamento de Prehistoria y Arqueología. Universidad Autónoma de Madrid. Email: mario.lopezr@estudiante.uam.es \\ 2 Departamento de Geología. Escuela Politécnica Superior de Ávila. Universidad de Salamanca \\ 3 Departamento de Edafología. E.T.S.I. Agrónomos. Universidad Politécnica de Madrid \\ 4 Departamento de Ciencias de la Tierra. Universidade do Coimbra \\ 5 Departamento de Arqueología, Paleontología y Recursos Culturales. Auditores de Energía y Medio Ambiente, S.A. \\ 6 Departamento de Historia y Filosofía, Área de Prehistoria. Universidad de Alcalá \\ 7 Laboratorio de Estratigrafía Biomolecular de la Escuela Técnica Superior de Ingenieros de Minas y Energía. Universidad \\ Politécnica de Madrid \\ 8 Nordic Laboratory for Luminescence Dating, Aarhus University / Radiation Research Division, Risø DTU, Denmark
}

\section{RESUMEN}

Se presentan los primeros resultados de los proyectos de investigación dirigidos al análisis cronoestratigráfico de las terrazas del río Tajo en el entorno de Toledo. El estudio se ha centrado en niveles fluviales del Pleistoceno Medio y Superior con yacimientos paleontológicos y achelenses con valoración tecnológica general similar al conjunto lítico de Pinedo (colección Máximo Martín Aguado). En el presente trabajo se incluyen las dataciones por IRSL y AAR de los niveles de terraza de $+25-30 \mathrm{~m}$ que contienen los yacimientos achelenses de Pinedo y Cien Fanegas. Las edades IRSL obtenidas (>280 ka y $292 \pm 17 \mathrm{ka}$ ) indican que la terraza y los yacimientos que contienen se formaron durante los estadios isotópicos MIS 9 y/o MIS 8, siendo correlacionables con la Unidad Arganda II en el valle del Jarama, aunque Pinedo podría representar parcialmente a la Unidad superior de Arganda I (MIS 11-MIS 9). La datación AAR obtenida para la Unidad superior de Pinedo indica una edad de $226 \pm 37$ ka. Aunque esta edad ha de tomarse con precauciones debido a la escasa contextualización estratigráfica y los bajos niveles de dentina de las muestras analizadas, podría indicar que el depósito de la terraza estudiada continuó hasta bien entrado el MIS 7, como también parece suceder en el Jarama (Valdocarros). En el tramo del valle analizado los procesos de sobre-sedimentación ligados a los procesos de subsidencia de las evaporitas del Neógeno (aguas arriba) y la existencia del nivel de base local impuesto por el Meandro Encajado de Toledo (aguas abajo), favorecieron los procesos de agradación fluvial sobre los de encajamiento. El método de datación utilizado (post IR-IRSL) ha permitido obtener la edad más antigua hasta la fecha sobre sedimentos fluviales arenosos en la Cuenca del Tajo.

Palabras clave: Geocronología; OSL-IRSL; AAR; terrazas fluviales; Achelense; Valle del Tajo; España Central.

Recibido el 26 de junio de 2014 / Aceptado el 20 de febrero de 2015 / Publicado online el 13 de mayo de 2015

Citation / Cómo citar este artículo: López-Recio, M. et al. (2015). Geocronología de los yacimientos achelenses de Pinedo y Cien Fanegas (Valle del Tajo) e implicaciones en la evolución fluvial en el entorno de Toledo (España). Estudios Geológicos 71(1): e029. http://dx.doi.org/10.3989/egeol.41816.340.

Copyright: () 2015 CSIC. This is an open-access article distributed under the terms of the Creative Commons Attribution-Non Commercial (by-nc) Spain 3.0 License. 


\section{ABSTRACT}

This study presents the first geochronological data from several research projects focused on the chronostratigraphy of the Tagus fluvial terraces in the environs of Toledo city (Central Spain). The research is centered on Middle to Late Pleistocene fluvial terraces with palaeontological and Acheulean sites of similar Palaeolithic technology than those showed by the lithic assemblage of the Pinedo site (Máximo Martín Aguado Collection). This study includes the first IRSL and AAR datings for the fluvial levels at $+25-30 \mathrm{~m}$ present in the Acheulean sites of Pinedo (Toledo) and Cien Fanegas (Aranjuez). The obtained ISRL dates (>280 ky and $292 \pm 17 \mathrm{ky}$ ) indicate that these fluvial levels and hence their associated Palaeolithic sites, were formed during the marine isotopic stages MIS 9 and MIS 8, and thus it is possible to correlate them with the Arganda II Unit of the adjacent Jarama valley. However, data from Pinedo suggest that this Acheulean site probably also correspond to the upper Arganda I Unit of the Jarama valley (MIS 11-MIS 9). Although the AAR date (226 $\pm 37 \mathrm{ky})$ obtained for the Upper unit of Pinedo has to be carefully considered (due to the scarce stratigraphic data and the low dentine levels found in the Equus sp. analyzed samples), it could indicate that sedimentation of the studied terrace level reached the MIS 7, as may also occur in the Jarama valley (Valdocarros site). In the analyzed sector of the Tagus valley, continuous fluvial sedimentation was favored by the occurrence of Neogene evaporites karst-linked subsidence (upstream) and the presence of an outstanding internal baselevel represented by the bedrock incised meander of Toledo (downstream). The dating method used in this study (post-IR- IRSL) has allowed us to obtain the oldest date from fluvial sandy deposits in the Tagus fluvial basin up to the present time.

Keywords: Geochronology; OSL-IRSL; AAR; Fluvial Terraces; Acheulean; Tagus Valley; Central Spain.

\section{Introducción}

Las terrazas del Tajo en el entorno de Toledo presentan interesantes yacimientos arqueológicos y paleontológicos documentados desde principios del siglo XX. Su geocronología se ha establecido clásicamente en base a la correlación de secuencias de terrazas, secuencias edáficas y los restos faunísticos asociados (Alférez, 1977; 1978; Pérez-González, 1994; Roquero et al., 1999). Por el contrario, las dataciones numéricas son prácticamente inexistentes, y sólo en los últimos años se han publicado datos al respecto en secuencias sedimentarias vinculadas a los niveles de terrazas de $+40 \mathrm{~m} \mathrm{y}+35 \mathrm{~m}$ aguas abajo de Toledo (Rodríguez de Tembleque, 2008; Ruiz Zapata et al., 2009). Hasta la fecha, los únicos datos paleomagnéticos disponibles correspondientes a la terraza de $+60 \mathrm{~m}$ (Salchicha), registran el tránsito entre el Pleistoceno Inferior y el Pleistoceno Medio (c. $780 \mathrm{ka}$ ) (Pinilla et al., 1995). Por ello, entre los años 2010 y 2011 se desarrolló el proyecto de investigación "Estudio cronoestratigráfico de las terrazas del Pleistoceno Medio y Superior para la reconstrucción paleoambiental del valle del río Tajo (Toledo)".

En el entorno de Toledo, el valle del Tajo reúne varios condicionamientos anómalos: a) el "Meandro Encajado de Toledo", del cual el río recibe su nombre (Tajo de Toledo; Hernández-Pacheco, 1930; Martín Aguado, 1990) y genera un represamiento natural del río aguas arriba, desde al menos el final del Pleistoceno Medio; b) Esta anomalía geomorfológica produce un fenómeno de sobre-sedimentación aguas arriba del meandro encajado, especialmente visible en los niveles de terraza e inferiores a $+30-35 \mathrm{~m}$, que ha facilitado la preservación de importantes yacimientos; c) la existencia de una falla inversa al sur de la ciudad que pone en contacto los materiales metamórficos de los Montes de Toledo con el relleno sedimentario de la Cuenca del Tajo y que afecta a los niveles de terrazas más altos $(>+70 \mathrm{~m})$ y pre-data el proceso de encajamiento anómalo.

Por otro lado, aguas arriba de Toledo (Añover de Tajo-Aranjuez), se produce el registro de la secuencia de terrazas casi al completo del valle, hecho que ha permitido el establecimiento de secuencias fluviales y catenas edáficas representativas del Pleistoceno para el sector central de la Península Ibérica (Roquero et al., 1999; 2015). Este sector participa de los fenómenos de engrosamiento de los depósitos fluviales característicos de los valles del sector central de la Cuenca del Tajo (Panera et al., 2011; Silva et al., 2013; Wolf et al., 2013) mantenido por los procesos de subsidencia kárstica que se producen inmediatamente aguas arriba y por el efecto de sobre-sedimentación (cola de embalse) que induce el Meandro encajado de Toledo aguas abajo. Estos condicionantes hacen que los niveles de terraza inferiores sujetos a mayores tasas de sedimentación 
y mayor potencia preserven significativos yacimientos paleolíticos y paleontológicos, como los analizados en este estudio (Pinedo y Cien Fanegas). En concreto, el yacimiento de Pinedo se encuentra apenas $1,5 \mathrm{~km}$ aguas arriba del meandro encajado. Cien Fanegas se localiza en una posición intermedia, $14 \mathrm{~km}$ aguas arriba de Toledo y unos $11 \mathrm{~km}$ aguas abajo de los últimos afloramientos en superficie de los materiales evaporíticos que provocan los procesos de subsidencia en el sector central de la Cuenca de Madrid, conjugándose el "efecto cola de embalse" del meandro encajado y el "efecto de mantenimiento de nivel de base" que procuran los fenómenos de subsidencia aguas arriba. De hecho es a partir de esta zona donde los niveles de terraza del Tajo inferiores a +30 m comienzan a presentar signos de engrosamiento, de hasta 9-10 m en Cien Fanegas, que no presentan los niveles fluviales superiores.

El principal objetivo del proyecto de investigación mencionado anteriormente se centró en la datación mediante distintos métodos de Luminiscencia de varios niveles de terrazas del Tajo en las inmediaciones de Toledo, utilizando OSL (Optically Stimulated Luminescence) en cuarzo (quartz-OSL) y también por pIRIR (post-IR Infrared Stimulated Luminescence) usando feldespato-K como dosímetro (Buylaert et al., 2012). La investigación se centró en aquellas terrazas con yacimientos paleontológicos y paleolíticos conocidos, con el fin de poder correlacionar los procesos de incisión-sedimentación fluviales con los diferentes estadios isotópicos (OIS) del Pleistoceno Medio y Superior y sus relaciones con la ocupación humana del territorio. Dadas las limitaciones geocronológicas de los métodos de datación utilizados (Buylaert et al., 2012), los análisis se realizaron en niveles fluviales inferiores a los $+40 \mathrm{~m}$ en los yacimientos de Pinedo y Cien Fanegas, aunque se ensayó la datación pIRIR en yacimientos situados a cotas relativas más altas $(+40$ y $+60 \mathrm{~m})$, como es el caso de los yacimientos de Valdelobos y Salchicha, actualmente en análisis (Fig. 1).

\section{Secuencia cronoestratigráfica de las terrazas del Tajo}

El área de estudio se sitúa en la Submeseta Sur peninsular, dentro de la cuenca cenozoica del Tajo, encuadrándose en su sector meridional (Cuenca Neógena de Madrid). De forma más concreta se localiza dentro del curso medio del río Tajo en el entorno de la ciudad de Toledo. Este río se ha ido encajando y generando sus depósitos fluviales a favor de la falla o contacto estructural que separa los dos dominios geológicos que constituyen el sustrato geológico del entorno de Toledo. Al sur se encuentran los materiales que componen la vertiente septentrional de

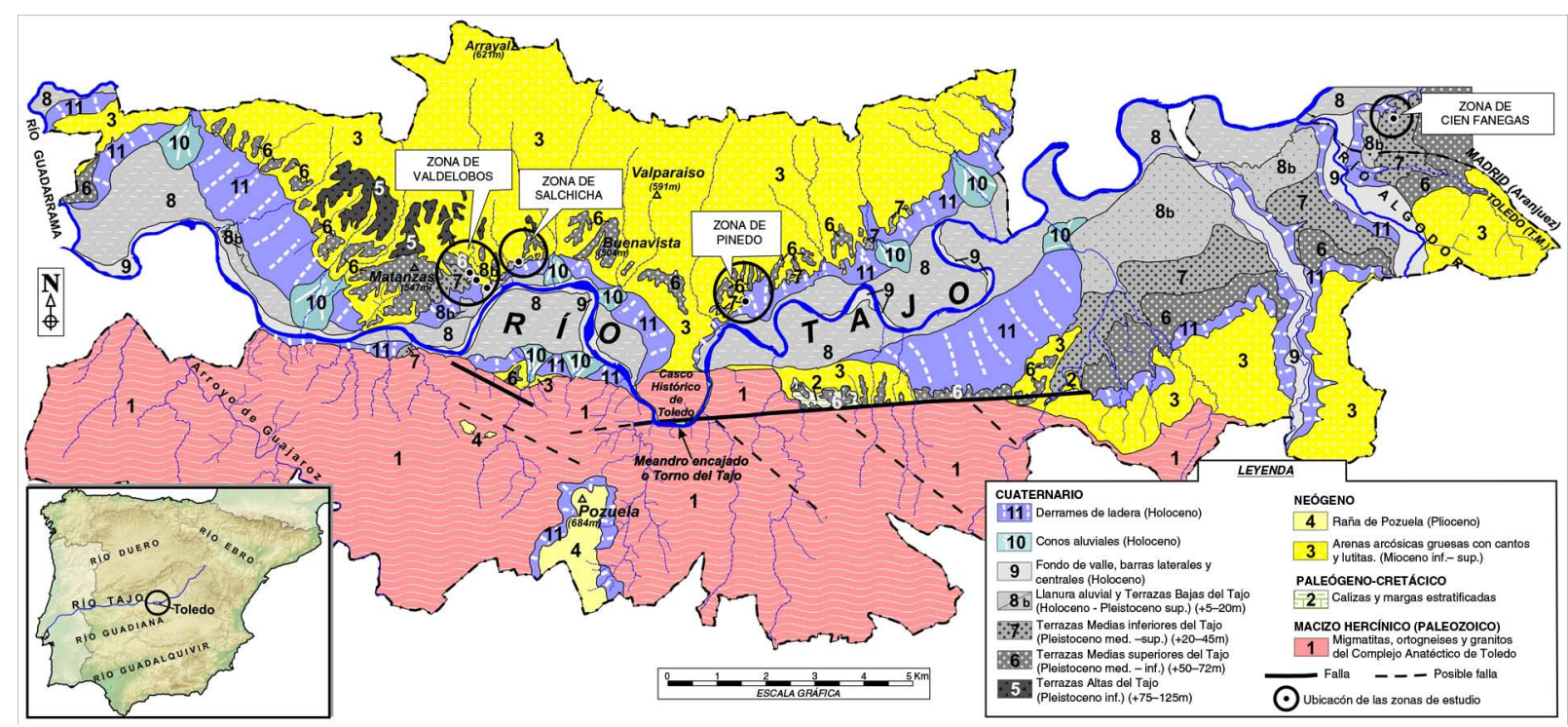

Fig. 1.-Localización geográfica y geológica de los yacimientos de Pinedo y Cien Fanegas, objeto de estudio, así como los yacimientos de Salchicha y Valdelobos citados en el texto (modificado de Herrero Matías, 1988 e IGME, en prensa). 
los Montes de Toledo dentro del Macizo Hercínico, mientras que al norte y este se localizan las arenas arcósicas que constituyen el relleno de la Cuenca neógena de Madrid. Tras la colmatación de la cuenca se generaron durante las etapas más tempranas de la sucesión climática cuaternaria amplias superficies de arrasamiento sobre las arenas arcósicas miocenas de las Facies Madrid o Toledo, depositadas con anterioridad (Pérez-González, 1982 y 1994). La continuada incisión de los incipientes cursos fluviales dará lugar al desmantelamiento de estas superficies o "Rampas arenofeldespáticas" (Vaudour, 1979), cuyos retazos han funcionado como divisoria de aguas hasta la actualidad.

En el entorno de la ciudad de Toledo se han llegado a diferenciar hasta trece niveles de terraza con cota relativa respecto al cauce (Pérez-González, 1994; Roquero et al., 1999; Pérez-González et al., 2004): +3-5 m (T13, llanura aluvial holocena); +4-9 m (T12, Peraleda y Arenero de Valdelobos) para el Pleistoceno Superior; +15-20 m (T11, Valdepastores, Villamejor y Monterrey), +25-30 m (T10, Pinedo y Cien Fanegas), $+40 \mathrm{~m}$ (T9, Salchicha y Buenavista inferiores-Campo de Tiro, Polígono Industrial), $+50 \mathrm{~m}$ (T8) y +60 m (T7, Buenavista, Salchicha y Valdelobos superiores) para el Pleistoceno Medio (Fig. 2); +75 m (T6, Salto de la Zorra), +85 m (T5), +95 m (T4, Bravo), +105 m (T3, Matanzas), +110-115 m
(T2) y +125 m (T1, Superficie de Bargas) para el Pleistoceno Inferior. Esta última superficie funciona como divisoria de aguas entre los valles del Tajo y Guadarrama, considerándosela como la superficie de referencia del encajamiento fluvial en la zona.

La terraza de $+60 \mathrm{~m}$ (Salchicha Superior) ha sido datada por paleomagnetismo (Pinilla et al., 1995), registrándose en ella la inversión Brunhes-Matuyama (780 ka). Ésta marca el límite Pleistoceno InferiorMedio y a nivel regional también se registra en terrazas de similar altimetría $(+60-55 \mathrm{~m})$ de otros valles de las cuencas del Tajo y Duero (Pérez-González et al., 2013). El resto de dataciones existentes en las inmediaciones del sector estudiado del valle del Tajo hacen referencia a formaciones aluviales posteriores al desarrollo de las terrazas, que las postdatan pero no son adecuadas para establecer una cronosecuencia fluvial fiable (Rodríguez de Tembleque, 2008; Ruiz Zapata et al., 2009).

\section{Contexto arqueológico}

\section{Las investigaciones paleolíticas en el valle medio del Tajo}

El estudio del Paleolítico en el valle medio del Tajo ha carecido hasta fechas recientes de proyectos de investigación dirigidos a la sistematización de

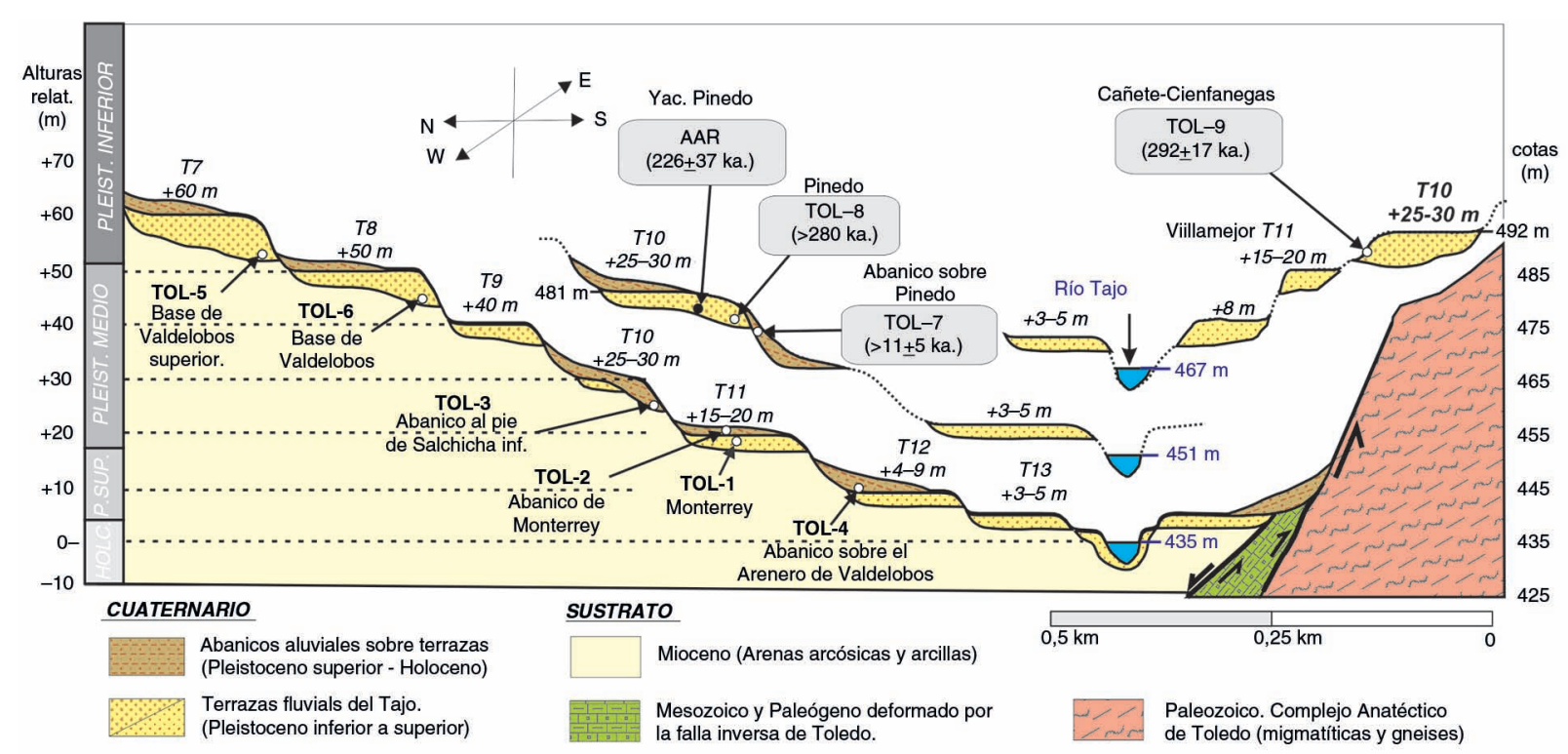

Fig. 2.-Corte geológico idealizado de los niveles de terrazas del Tajo a la altura de Toledo, con los resultados de las dataciones del presente estudio. 
la ocupación paleolítica, basándose principalmente en hallazgos aislados, desprovistos de contextualización geológica, diseminados principalmente por las terrazas fluviales del Tajo y sobre la superficie de formaciones plio-cuaternarias (rañas) y cuaternarias (glacis, coluviones, abanicos aluviales, etc.). Las primeras referencias sobre hallazgos paleolíticos en terrazas del valle medio del Tajo se remontan al primer tercio del s. XX, momento en el que se efectuaron los descubrimientos de fauna pleistocena e industria lítica en Mesegar de Tajo, en la mitad occidental de la provincia (De Combes, 1906), y la presencia de utensilios paleolíticos en los alrededores de Toledo, concretamente en los parajes de Buenavista y Valdecubas (Pérez de Barradas, 1920), corroborado posteriormente por I. del Pan (1928).

A mediados del s. XX, los trabajos de Martín Aguado $(1962 ; 1963)$ constituyeron un revulsivo para el estudio de las terrazas del Tajo en los alrededores de Toledo. Este investigador, por un lado, descubrió en una gravera de la finca de Buenavista restos de un Palaeoloxodon antiquus, del cual pudieron recuperarse dos defensas y los molares superiores, y por otro lado, documentó en las graveras de la finca de Pinedo $(T+25-30 \mathrm{~m})$ la presencia de industria paleolítica en posición estratigráfica. Estos hallazgos motivaron que dicho investigador iniciara una labor prospectiva minuciosa de los frentes de las graveras abiertas del río próximas a la ciudad de Toledo. Paulatinamente fueron encontrándose nuevos conjuntos líticos achelenses, así como nuevos hallazgos paleontológicos, sobre todo obtenidos en la gravera de Pinedo: Palaeoloxodon antiquus, Hippopotamus, Rhinoceros, Cervus, Bos y Equus (Aguirre, 1965).

Pinedo fue el primer yacimiento achelense excavado de forma sistemática en la zona central de la Península entre 1972 y 1976 (Querol \& Santonja, 1979). Durante el período de excavación de dicho yacimiento, estos investigadores realizaron prospecciones en otros puntos de las terrazas del río Tajo, con el objetivo de conseguir referencias cronoestratigráficas válidas para el conjunto del valle. De este modo se localizaron industrias líticas achelenses primitivas, muy dudosas, en Talavera de la Reina, El Espinar (Almonacid, Toledo) y Polígono Industrial de Toledo (Toledo) (Santonja, 1981; Santonja \& Querol, 1982). Aguas arriba de Toledo, en la gravera de Cien Fanegas $(\mathrm{T}+25-30 \mathrm{~m})$ se recogieron piezas achelenses, asimilables a las de Pinedo, junto a restos de macrovertebrados del Pleistoceno Medio (Rus et al., 1993). Más recientemente destaca la excavación del yacimiento achelense de Puente Pino $(\mathrm{T}+40 \mathrm{~m})$ situado en Alcolea de Tajo, aguas abajo de Toledo (Rodríguez de Tembleque et al., 2005; Ruiz Zapata et al., 2009).

\section{El Achelense en el valle medio del Tajo}

Los primeros indicios de ocupación humana de la cuenca media del río Tajo se remontan al Pleistoceno Inferior. De este modo, en el entorno de Toledo existen indicios de industria lítica en El Espinar (Santonja, 1981; Santonja \& Querol, 1982), Salto de la Zorra y Santa María de Benquerencia, situados en la terraza de $+75-80 \mathrm{~m}$ (Rodríguez de Tembleque et al., 2010). La industria hallada en posición estratigráfica se reduce a elementos aislados, que se asemejan a los conjuntos achelenses (Rodríguez de Tembleque et al., 2010) y que resultan sorprendentes a la vez que significativos en relación con la presencia humana en la región desde el Pleistoceno Inferior. La macrofauna documentada de Equus stenonis en Salto de la Zorra (T +75 m; Alférez, 1978; Sesé et al., 2000) y Mammuthus meridionalis en Salchicha (+60 m; Sesé et al., 2000) indican una edad Pleistoceno Inferior para estos niveles fluviales. Aunque no se dispone de dataciones númericas para estos niveles fluviales altos, dataciones ESR de la terraza de $+70-75 \mathrm{~m}$ en la cuenca del Duero (valle del Arlanzón) indican que esta terraza se desarrolló en torno a 1 M.a. dentro del Subchron Normal Jaramillo (Moreno et al., 2012), datos que concuerdan con las determinaciones paleomagnéticas realizadas en depósitos fluviales asimilables a esta terraza en el valle del Jarama (Pérez-González et al., 2013). Como ya se ha comentado, los datos paleomagnéticos correspondientes a la $\mathrm{T}+60 \mathrm{~m}$ en el valle del Tajo situan en este nivel el tránsito Pleistoceno Inferior-Medio (780 Ka; Pinilla et al., 1995).

Yacimientos achelenses, con industria lítica compuesta por macroutillaje (bifaces, triedros, hendedores) y lascas de gran formato, se atestiguan en las terrazas medias ( $\mathrm{T}+40$ a $\mathrm{T}+20 \mathrm{~m}$ ) del Tajo (Santonja \& Pérez-González, 2002; Santonja \& Villa, 2006). En Toledo se localizó industria lítica en la $\mathrm{T}+40 \mathrm{~m}$ 
(Polígono industrial) asociada estratigráficamente a restos de Mammuthus trogontherii (Sesé et al., 2000). Además de M. trogontherii, la lista faunística de la $\mathrm{T}+40 \mathrm{~m}$ en los yacimientos de Buenavista inferior, Salchicha inferior y Polígono industrial, comprende Equus caballus, Hippopotamus amphibius, Dolichodoriceros savini, Eliomys quercinus, Allocricetus bursae, Microtus brecciensis y Apodemus cf. Sylvaticus, todos ellos característicos del Pleistoceno Medio (Sesé et al., 2000).

A su vez, destacan los yacimientos achelenses de Cañete Chico y Cañete Grande, en Villasequilla de Yepes (Santonja, 1981) y Cien Fanegas al Sureste de Aranjuez (Rus et al., 1993; Baena et al., 2010b), ambos correlacionables con la terraza de Pinedo $(\mathrm{T}+25-30 \mathrm{~m})$. Estos yacimientos cuentan con conjuntos líticos en cuarcita local procedente de los depósitos de la terraza. Del mismo modo, en las inmediaciones de Toledo se han localizado restos faunísticos del Pleistoceno Medio en las terrazas de Buenavista, Valdelobos, Valdecubas, Campo de Tiro, Observatorio y El Pocito (Alférez, 1977).

Pinedo es el yacimiento del Pleistoceno Medio más representativo de los conocidos en la zona, situado en la terraza de $+25-30 \mathrm{~m}$ del Tajo, inmediatamente aguas arriba de Toledo. En la excavación de $25 \mathrm{~m}^{2}$ efectuada en la base de dicha terraza (Querol $\&$ Santonja, 1979) se detectó un importante conjunto lítico removilizado, contextualizado en niveles de arenas y gravas (Santonja, 1992). Los niveles basales de dicho yacimiento también contenían restos faunísticos correspondientes a Lepus cf. europaeus, Oryctolagus cuniculus, Equus sp., Cervus elaphus, Bos sp., Elephas antiquus, Hippopotamus amphibius y Praedama cf. süssenbornensis (Soto, 1979), fauna representativa del Pleistoceno Medio final (Sesé et al., 2000).

\section{Rasgos tecnológicos del conjunto lítico de Pinedo}

Según Querol y Santonja (1979), tras la excavación sistemática del yacimiento, las características generales de la industria achelense son las siguientes: destaca el empleo de la cuarcita como materia prima (68\%) frente al menor empleo del sílex (25\%) y el cuarzo (7\%), materias primas transportadas por el río Tajo. Existe un aprovechamiento casi exclusivo de cantos rodados de cuarcita de transporte fluvial, el índice levallois técnico es nulo y el índice de facetado es muy bajo y los núcleos son de gran tamaño, espesos y con escasas extracciones. Por otro lado, existe un elevado porcentaje y diversidad de instrumentos sobre lasca, con predominio de raederas y cuchillos de dorso. Dentro del macroutillaje los bifaces más frecuentes son los amigdaloides (18\%), lanceolados (15\%) y abbevillenses; existen 38 hendedores, en su mayoría de tipos primitivos (Tipo 0 , I y II) y destaca el porcentaje de triedros $(8 \%)$, siendo superior al de bifaces y hendedores.

Tras la revisión e inventario por nuestra parte de los materiales líticos de Pinedo de la colección Máximo Martín Aguado, se clasificaron desde el punto de vista morfotécnico 6868 piezas líticas correspondientes a dicho yacimiento (Tabla 1), con especial atención a la caracterización de los procesos de façonnage y débitage. Destaca la representatividad del conjunto, si bien se trata de una recogida sesgada y dirigida de los materiales, tanto en su propio depósito natural, como en las labores

Tabla 1.-Tabla de clasificación morfotécnica del conjunto lítico de Pinedo (Colección Martín Aguado)

\begin{tabular}{|c|c|}
\hline CATEGORÍA MORFOTÉCNICA & $\mathbf{N}^{\circ}$ \\
\hline Lasca/frag. & 2104 \\
\hline Bifaz/bifaz parcial & 1527 \\
\hline Canto trabajado/frag. & 1523 \\
\hline Triedro/pico triédrico /frag. & 750 \\
\hline Núcleo/frag. & 304 \\
\hline Protobifaz & 288 \\
\hline Indeterminado/frag. & 175 \\
\hline Hendedor & 56 \\
\hline Raedera & 47 \\
\hline Denticulado & 30 \\
\hline Unifacial & 25 \\
\hline Resto de talla & 23 \\
\hline Nódulo & 10 \\
\hline Cuchillo de dorso natural & 2 \\
\hline Bola/esferoide & 1 \\
\hline Cepillo & 1 \\
\hline Muesca & 1 \\
\hline Perforador & 1 \\
\hline Total & 6868 \\
\hline
\end{tabular}


de recogida. A pesar de las limitaciones interpretativas que presenta esta colección, la procedencia de los materiales puede guardar una cierta coherencia a nivel arqueoestratigráfico con el conjunto procedente de los niveles excavados (Querol \& Santonja, 1979). Destaca también el elevado número de lascas de gran formato, cantos tallados, bifaces, triedros (Fig. 3C) y hendedores, así como núcleos de escasas extracciones y de débitage bifacial. De este modo, se ha constatado que la configuración general del macroutillaje parece decantarse de manera intensa hacia la confección de morfologías triangulares. En la mayor parte de las piezas analizadas existen algunos rasgos morfológicos que parecen condicionar la selección de los soportes. Muchas de las piezas son configuradas mediante façonnage a partir de grandes lascas obtenidas de cantos que presentan a su vez morfologías específicas (formas oblongas o tendencia a las secciones cuadrangulares, pero siempre con escaso espesor). Por tanto, se constata que existió una selección de morfologías de cantos próximas al producto buscado, o bien una selección de cantos con morfologías que permitieran la obtención de lascas de formatos próximos al producto buscado.

Los esquemas diacríticos indican que el proceso de configuración se establece generalmente en series de 2 ó 3 extracciones contiguas, de carácter no alternante sobre cada uno de las caras talladas, creando con ello una morfología de sección triangular que responden a esquemas de configuración o façonnage que se repiten sistemáticamente (Fig. 3B). Rasgos tecnológicos similares han sido descritos para los cercanos yacimientos de Tafesa (Baena \& Baquedano, 2004; Baena et al., 2010a), Puente Pino (Rodríguez de Tembleque, 2008; Rodríguez de Tembleque et al., 2005) o en el Cortijo del Calvillo en Granada (Jiménez-Cobos, 2012).

Con características tecnológicas similares, el conjunto lítico de Cien Fanegas (Aranjuez), compuesto por solo 130 piezas, cuenta con un número elevado de triedros, bifaces y hendedores. Presenta un façonnage de esquemas tecnológicos sencillos, con escasa alternancia entre las series de extracciones (Fig. 3A), así como lascas corticales y semicorticales y núcleos expeditivos de escasa programación (multipolar), existiendo una estrategia de selección de soportes con buena calidad para la talla, y de soportes próximos a la morfología del producto final (Rus et al., 1993; Baena et al., 2010b).

\section{Metodología}

Se ha obtenido un número significativo de dataciones por quartz-OSL y también por pIRIR en distintos niveles de terrazas del Pleistoceno Medio y Superior situadas entre la llanura aluvial y el nivel $\mathrm{de}+60 \mathrm{~m}$. Las fracciones minerales OSL fueron preparadas en el Dep. Ciências da Terra-Univ. Coimbra y la medición OSL en el Nordic Laboratory for Luminescence Dating (NLL). Inicialmente se realizó una datación OSL convencional en cuarzo (Murray \& Olley, 2002). Pero, debido a que las tasas de radiación ambiental (dose rates) de los sedimentos eran relativamente altas, la señal del OSL en cuarzo resultó estar saturada para todas las muestras excepto para las más recientes. Por tanto, se empleó el método post-IR IRSL (pIRIR; post-Infrared Stimulated Luminiscence) que permite la datación de muestras de mayor antigüedad (Buylaert et al., 2009, 2012). El método, cuyos protocolos detallan los trabajos citados, consiste en la estimulación de la termoluminiscencia de granos de cuarzo y feldespato potásico mediante radiación infrarroja a $250{ }^{\circ} \mathrm{C}$, mejorando las determinaciones de las tasas de radiación ambiental (dose rates) para depósitos en los que los métodos convencionales OSL en cuarzo resultan estar saturados o al límite de la saturación. En nuestro caso el método se aplicó sobre granos de feldespato K que según los protocolos de datación retienen una mejor señal de las dosis de radiación recibidas, sin llegarse a saturar (i.e. Buylaert et al., 2012). Este método también se empleó en niveles fluviales de $+60 \mathrm{~m},+50 \mathrm{~m} \mathrm{y}+40 \mathrm{~m}$, si bien en este trabajo solo se presentan los resultados de las dataciones del nivel $+25-30 \mathrm{~m}$.

Por otro lado, de forma complementaria, se procedió a la datación de tres molares de Equus por racemización de aminoácidos (AAR). La preparación de las muestras se realizó siguiendo los protocolos estándar del Laboratorio de Biología Molecular (LEB) de la Universidad Politécnica de Madrid (Torres et al., 2002), analizándose en un cromatógrafo de líquidos HPLC-1100 con detector de fluorescencia. La dentina se obtuvo perforando con una broca de diamante los molares analizados y posteriormente se dializaron con el fin de determinar los 


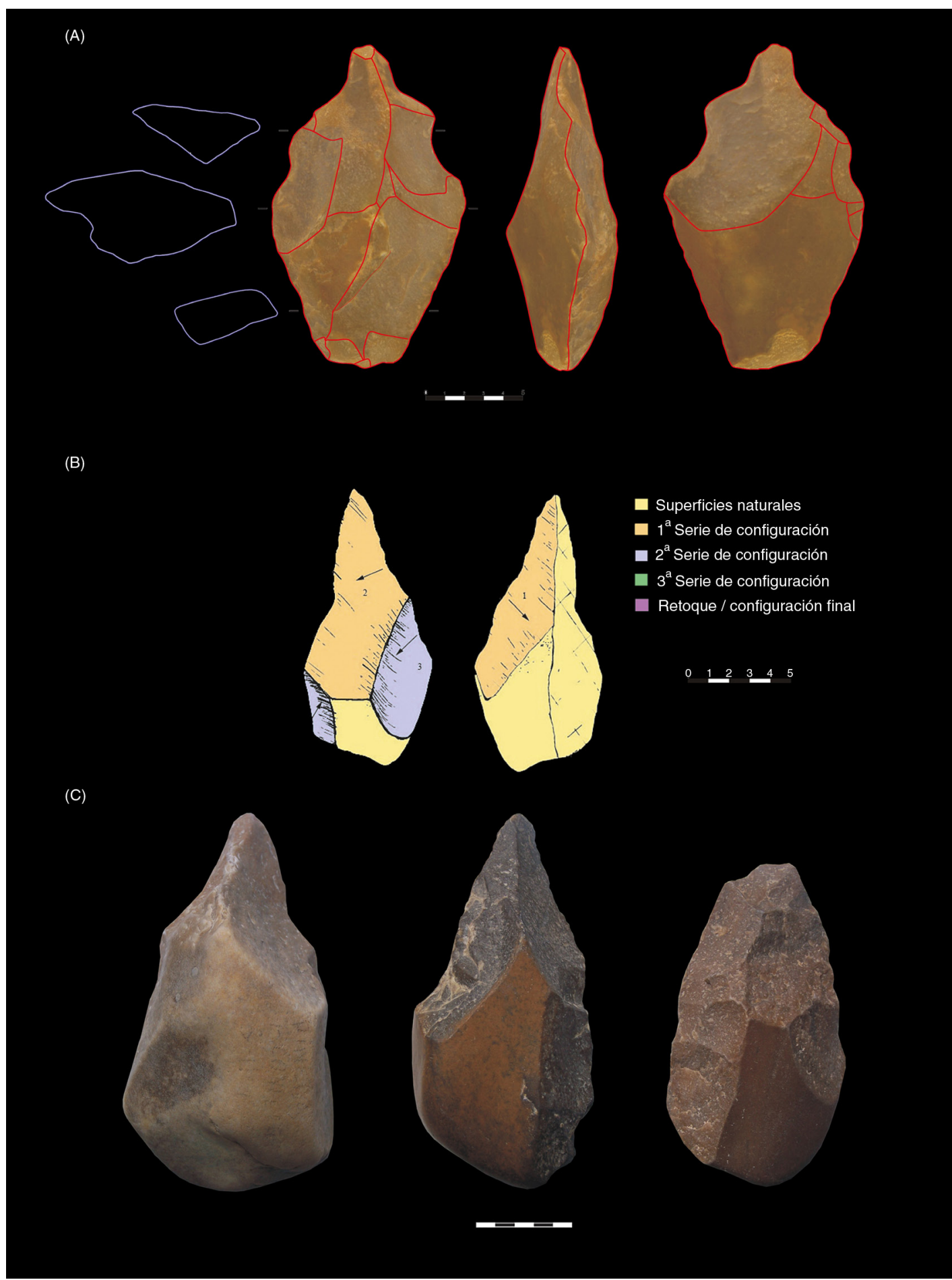

Fig. 3.-A) Representación 3D de un bifaz de Cien Fanegas (F.J. López Fraile); B) Esquema diacrítico de triedro de Pinedo (Colección Martín Aguado); C) Triedros y bifaz de Pinedo (Colección Martín Aguado).

aminoácidos libres que pudieran interferir en su posterior análisis (Torres et al., 2002). La edad se determinó introduciendo los valores $\mathrm{D} / \mathrm{L}$ del ácido aspártico en el algoritmo de cálculo de edad establecido para el colágeno de la dentina de osos fósiles de la Península Ibérica, tales como Ursus deningeri 
y Ursus spelaeus (Torres et al., 2014). A pesar de que el proceso de racemización es variable en cada especie, se ha comprobado que las diferencias de racemización en el colágeno de mamíferos no son significativas para su datación (Torres et al., 2002). De hecho esta metodología ya ha sido aplicada en la datación de piezas dentales de mamíferos (Equus, $B o s$, etc.) y herpetofauna fósil en yacimientos pleistocenos de la zona de Madrid (i.e. Panera et al., 2011; Blain et al., 2012).

En conjunto los datos que se desprenden de estos trabajos es que las técnicas convencionales TL/OSL quedan muy al límite de las edades correspondientes a los niveles fluviales $\geq+25 \mathrm{~m}$ (i.e Silva et al., 2013), siendo preciso la utilización de técnicas más refinadas (pIRIR) o acudir a otros métodos de datación (AAR) cuya resolución permite definir edades de cierta antigüedad aún a costa de márgenes de incertidumbre algo más elevados (Torres et al., 2014).

\section{Estratigrafía y cronología de la terraza de +25-30 m}

\section{Pinedo}

El afloramiento donde se ha documentado y muestreado el retazo de la terraza que albergaba el yacimiento de Pinedo se sitúa en el desmonte Norte del ramal de salida de la autopista AP-41 hacia la carretera CM-4001, en el entorno del perímetro urbano NE de la ciudad de Toledo. En este afloramiento se registra la base de la terraza de $+25-30 \mathrm{~m}$, constituida por gravas heterométricas de gran tamaño en contacto erosivo sobre las arenas arcillosas miocenas
(Fig. 4). El nivel basal de gravas posee una potencia de 2 a $3 \mathrm{~m}$ y se acuña progresivamente hacia el NE pasando lateralmente a lentejones de arenas medias y finas que se muestrearon para su datación OSL (TOL-8). El yacimiento excavado entre 1972 y 1974 se situaba en niveles de arenas y gravas basales de la terraza correspondientes a las Unidades Inferior y Superior de Díaz y Pérez-González (1979), que aquí denominaremos Pinedo I y Pinedo II respectivamente. Ambas unidades corresponderían a depósitos de barras y relleno de canal, con potencias de 4,40 m (Pinedo I) y de algo más de $1 \mathrm{~m}$ (Pinedo II), esta última de carácter más arenoso. Por encima de estos depósitos se situaba un paquete de $9 \mathrm{~m}$ de potencia (Pinedo III) constituido por una alternancia de arcillas y limos arcillosos rojizos que evoluciona lateralmente y en la vertical a arenas cuarzo-feldespáticas procedentes de los relieves miocenos adyacentes. La secuencia continuaba con una unidad de arenas coluviales con matriz limo-arcillosa $(3,5-4 \mathrm{~m})$ con un lag basal de gravas angulosas heterométricas, de hasta $0,8 \mathrm{~m}$ de potencia, en discordancia erosiva sobre las unidades anteriores (Pinedo IV). El lag basal de Pinedo IV contenía cantos blandos de un suelo rojo 2.5 YR 4/8 (Díaz \& Pérez-González, 1979), probablemente desarrollado a techo de la unidad infrayacente Pinedo III. Todo el conjunto se encontraba sellado por un nivel de limos de origen eólico fuertemente carbonatados de 1 a $1,5 \mathrm{~m}$ de potencia (Pinedo V). En total, los depósitos de la terraza sobrepasaban claramente los $17 \mathrm{~m}$ de potencia, pero tan solo los niveles basales de gravas y arenas (5,4 m; Pinedo I y II) eran de origen fluvial; las unidades superiores (Pinedo III y IV) eran

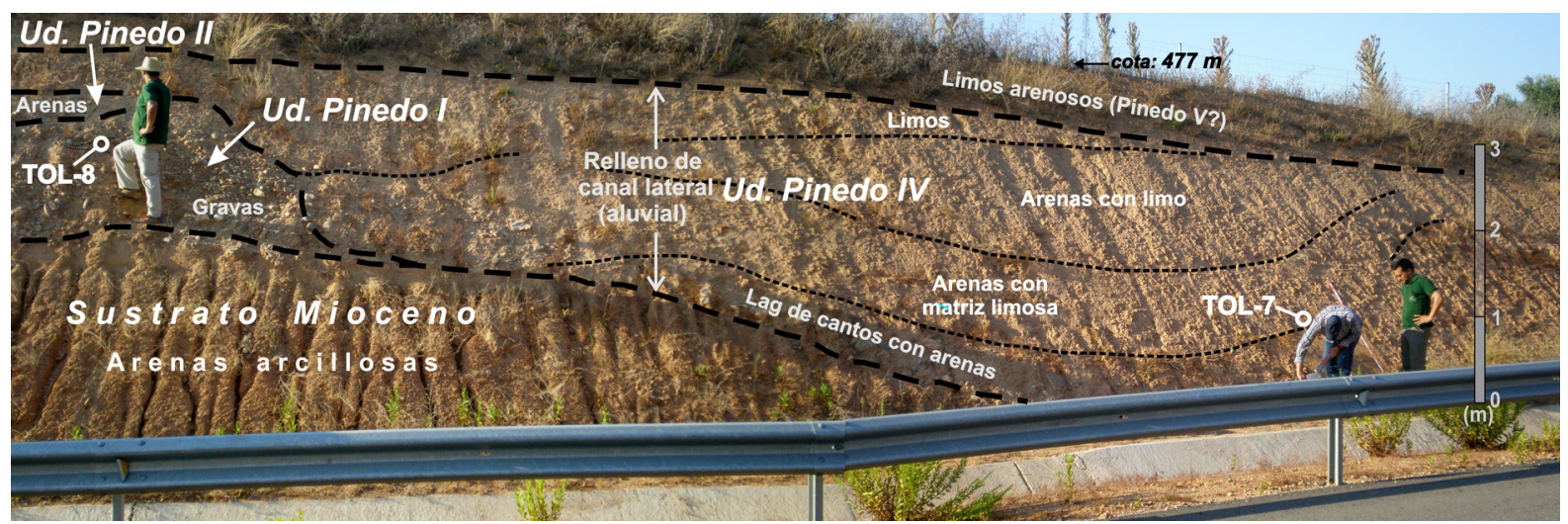

Fig. 4.-Estratigrafía de la formación Pinedo. 
de claro origen lateral y Pinedo IV, en discordancia erosiva, de origen más reciente. Estas se encontraban desmantelando los depósitos fluviales de Pinedo I y II, testificando la gran potencia de Pinedo III debido a los procesos de sobre-sedimentación asociados al represamiento natural del Tajo como consecuencia de la existencia del Meandro encajado de Toledo. Es importante señalar que los conjuntos líticos y paleontológicos del antiguo yacimiento de Pinedo se encontraban ligados a las unidades fluviales basales Pinedo I (el más importante) y Pinedo II (con restos más dispersos). En ambos casos los elementos líticos y faunísticos se encontraron en las facies de gravas y arenas con lo cual serían transportados y no "in situ" (Díaz y Pérez-González, 1979), lo que podría implicar que la edad de los elementos recuperados podría ser ligeramente anterior a la de la propia terraza.

En los afloramientos actuales, las gravas y arenas basales (Pinedo I y II) son muy restringidos, aunque alcanzan localmente potencias de 2-3 m (Fig. 4). Ambas unidades llegan a desaparecer erosionadas por aportes aluviales laterales de carácter arenoso y matriz limo-arcillosa, que poseen un significativo lag basal de cantos heterométricos de gran tamaño procedentes del desmantelamiento de la terraza. Esta formación aluvial secundaria presenta una significativa potencia (4-5 m), desarrollando amplios canales que erosionan las unidades inferiores, con lag basales de gravas de gran tamaño, y que interpretamos como la unidad Pinedo IV. En la secuencia actual no se observa la potente unidad Pinedo III documentada por Díaz y Pérez-González (1979), y la secuencia sedimentaria culmina con un nivel de limos arcillosos y arenas limosas de tonos anaranjados-rojizos de $0,5 \mathrm{~m}$ de potencia que presenta un débil desarrollo edáfico con pequeñas rizoconcreciones carbonatadas (Fig. 5), que quizá podría corresponder con la unidad eólica de Pinedo V o ser aportes más recientes.

Para el presente trabajo se muestrearon la parte basal arenosa, por encima del lag de gravas heterométricas, de Pinedo IV (TOL-7) y los niveles más arenosos de Pinedo I (TOL-8) tal y como se ilustra en la Fig. 4. Esta última muestra correspondería con la posición del antiguo yacimiento de Pinedo y por tanto la edad OSL obtenida sería representativa de la cronología del mismo.

\section{Cien Fanegas}

Del mismo modo, se procedió a estudiar y muestrear otros sectores del valle con cortes de esta terraza bien conservados. El más representativo lo constituye el yacimiento paleolítico del arenero de "Cien Fanegas", situado en la margen derecha del río Algodor en su zona de confluencia con el Tajo, aguas arriba de Toledo, en la terraza de $+25-30 \mathrm{~m}$. Se documentaron restos faunísticos del Pleistoceno Medio e industria achelense, similares a los de Pinedo en sus

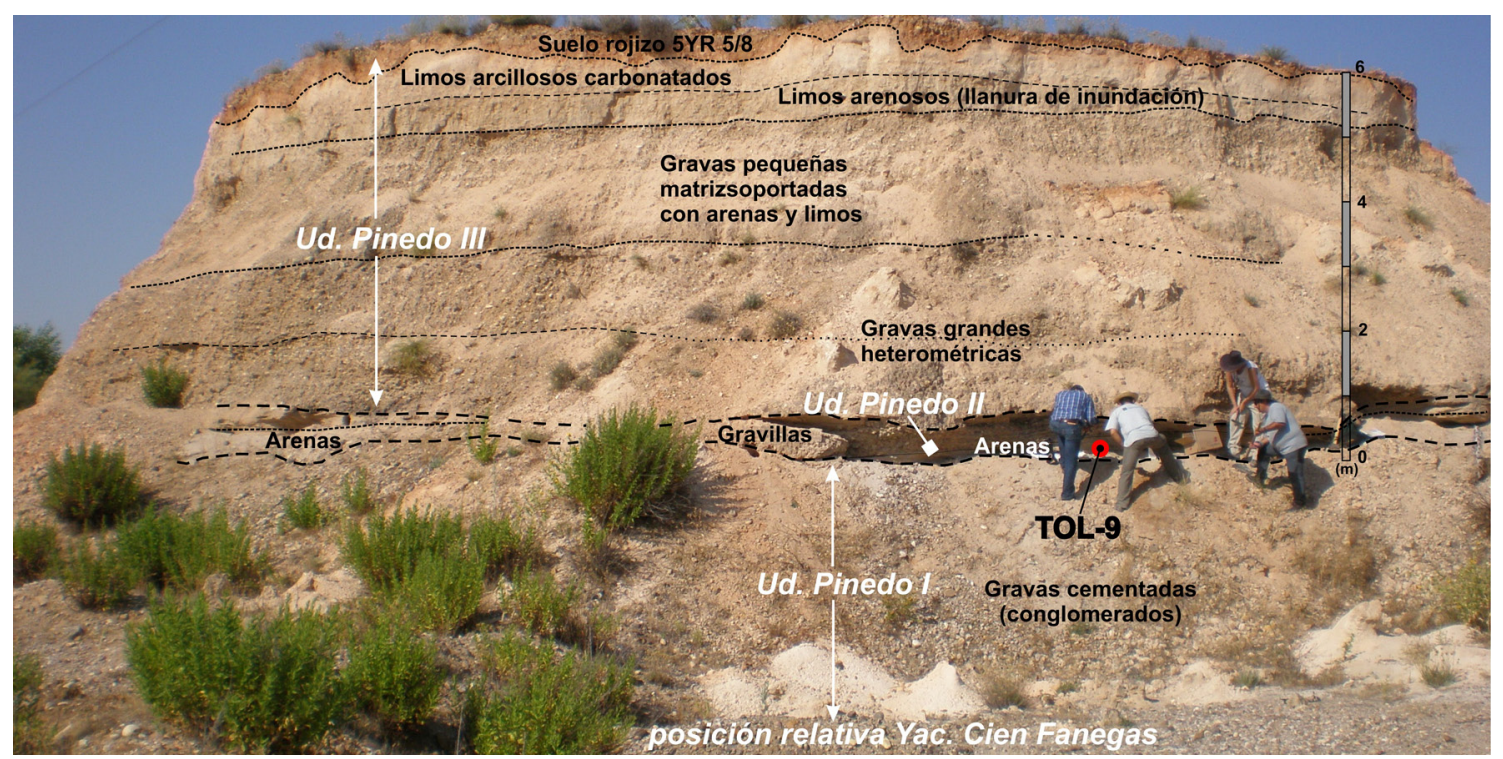

Fig. 5.-Estratigrafía del arenero de Cien Fanegas. 
niveles basales de gravas (Rus et al., 1993; Baena et al., 2010b). Los cortes actuales conservan $6 \mathrm{~m}$ de la secuencia fluvial de la terraza desde su zona basal, compuesta por niveles de gravas y arenas variablemente cementados, hasta su zona superior donde se desarrollan facies arcillo-limosas de llanura de inundación. Se han diferenciado dos grandes unidades que correlacionamos, dado su origen fluvial, con las unidades Pinedo I y Pinedo II, aunque las facies de llanura de inundación podrían representar y correlacionarse con Pinedo III.

Tal y como se observa en la Fig. 5 sobre el paquete arcillo-limoso de llanura de inundación se desarrolla un nivel de gravas pequeñas subangulosas matrizsoportadas, con matriz limo-arenosa de algo más de $2 \mathrm{~m}$ de potencia y podrían representar aportes laterales. Las facies de llanura de inundación propiamente dicha se sitúan a techo (1-1,2 $\mathrm{m}$ de potencia) y sobre ella se desarrolla un suelo haploxeralf cálcico de $70 \mathrm{~cm}$ de potencia con un horizonte $\mathrm{Bt}$ de color rojizo (5YR 5/8s -2.5 YR 4/8h) bien desarrollado (Fig. 5). Este suelo es similar al desmantelado en Pinedo (Pinedo III) e incluido como cantos blandos en el lag basal de gravas de la unidad erosiva más reciente Pinedo IV. En el arenero de Cien Fanegas todo el conjunto está fosilizado por un nivel superior arenoso de menos de $1 \mathrm{~m}$ de potencia que incluye cantos de cuarcita facetados, indicando que se trata de un nivel aluvial posterior a la terraza y con evidente retoque eólico, que podría correlacionarse con la unidad eólica de Pinedo V. En esta localidad se muestreó para OSL (TOL-9) un nivel de arenas con estratificación cruzada planar y de surco que se situaría a techo de la Unidad Pinedo I (Fig. 5), unos $2 \mathrm{~m}$ por encima del nivel de gravas basal donde se documentó la industria achelense en este yacimiento (Rus et al., 1993; Baena et al., 2010b).

\section{Cronología de la terraza $+25-30 \mathrm{~m}$}

En el presente trabajo nos centraremos en las dataciones obtenidas en el nivel de +25-30 m (T10) donde se sitúan los yacimientos de Pinedo (Querol \& Santonja, 1979) y Cien Fanegas (Rus et al., 1993).

Los resultados de las muestras tomadas en el yacimiento de Pinedo indican que la base de la terraza en esta zona (Pinedo I; TOL-8) es anterior a los $280 \mathrm{ka}$ (Pleistoceno Medio final) y que la unidad aluvial discordante que la erosiona en esta zona (Pinedo IV; TOL-7) posee una edad muy reciente de $11 \pm 0,9 \mathrm{ka}$ correspondiente al final del Pleistoceno Superior. La datación realizada en el techo de la unidad basal de la terraza (Pinedo I; TOL-9) del arenero de Cien Fanegas arroja una edad de 292 \pm 17 ka, encuadrándola en el tránsito de los estadios isotópicos marinos MIS 8 y MIS 9 (c. 300 ka; Imbrie et al., 1992). Los datos numéricos y analíticos de las dataciones efectuadas se presentan en la Tabla 2.

Complementariamente, se analizaron tres piezas dentales de Equus sp. provenientes de los niveles superiores de las gravas del yacimiento de Pinedo (Pinedo II) para su datación por Racemización de Aminoácidos (AAR; Tabla 3 y Fig. 6). En dos de las muestras se obtuvo polvo de dentina, y en otra de esmalte, con la ayuda de una microperforadora con broca de diamante en el Laboratorio de Estratigrafía Biomolecular de la Escuela Técnica Superior de Ingenieros de Minas de Madrid. El contenido de aminoácidos de las muestras BSL-4764 y BSL-4766 fue insuficiente para proceder a su datación; sin embargo la muestra BSL-4765 presentó un contenido mínimo, pero suficiente para proceder a su datación AAR. La pieza dentaria analizada (BSL-4765), un molar inferior de Equus sp., arrojó una edad de $226 \pm 37$ ka (Tabla 3 y Fig. 6), ciertamente más reciente que la datación por IRSL ( $>280 \mathrm{ka}$ ) obtenida en los sedimentos arenosos de Pinedo I y que nos situaría en el MIS 7 (Imbrie et al., 1992).

\section{Interpretación}

Las edades obtenidas cerca de la base de la terraza T +25-30 m del río Tajo en Cien Fanegas (TOL-9: $292 \pm 17 \mathrm{ka}$ ) sitúan la base de la terraza fluvial (Unidad Pinedo I) en un rango geocronológico comprendido entre 309 y $275 \mathrm{ka}$, correspondiente al tránsito MIS 9-MIS 8 (Fig. 7). Sin embargo la edad obtenida en la misma unidad en el propio yacimiento de Pinedo (TOL-8: >280 ka), sugiere que su depósito pudo comenzar antes, quizá desde el inicio del MIS 9 (c. $340 \mathrm{ka}$ ). Por otro lado, la edad AAR obtenida en Pinedo (226 $\pm 37 \mathrm{ka}$ ), probablemente procedente de la unidad superior (Pinedo II), indicaría de forma genérica que el depósito de la terraza en el entorno del Meandro encajado de Toledo pudo continuar hasta el MIS 7 (Fig. 7). 
Tabla 2.-Tabla de dataciones IRSL de Pinedo y Cien Fanegas

\begin{tabular}{llrrrrr}
\hline $\begin{array}{l}\text { Código NLL/ } \\
\text { Código campo }\end{array}$ & \multicolumn{1}{c}{$\begin{array}{c}\text { Posición geomorfológica } \\
\text { y estratigráfica }\end{array}$} & Edad (ka) & Equiv. Dose (Gy) & (n) & Dose rate (Gy/ka) & \% Agua \\
\hline $102258 / T O L-7$ & Aluvial al pie de la Terraza T 10 (+25-30m) & $11,0 \pm 0,9$ & $56 \pm 4$ & 6 & $5,12 \pm 0,19$ & 11 \\
$102259 / T O L-8$ & Base de la Terraza T 10 (+25-30m) & $>280$ & $>1000$ & - & $3,52 \pm 0,13$ & 10 \\
$102260 / T O L-9$ & Terraza T 10-11 (+20-25m) & $292 \pm 17$ & $960 \pm 38$ & 6 & $3,282 \pm 0,11$ & 10 \\
\hline
\end{tabular}

Las edades obtenidas se encuentran en el rango cronológico de las unidades Arganda I y Arganda II del valle del Jarama, obtenidas mediante análisis AAR (Panera et al., 2011; Blain et al., 2012). Los datos de estos autores indican que ambas unidades, superpuestas en la base de la terraza compleja de $+18-20 \mathrm{~m}$, presentan edades de c. 332-379 ka (Arganda I: MIS 11-MIS 9) y 254-262 ka (MIS 8) (Panera et al., 2011), y se asocian a yacimientos paleontológicos y achelenses, aunque sus conjuntos líticos presentan características morfotécnicas diferentes a los documentados en este trabajo. Ambas unidades son correlacionadas con los niveles de terraza a $+30 \mathrm{~m}$ (Arganda I) y a $+25 \mathrm{~m}$ (Arganda II) que aparecen escalonados en el Jarama aguas arriba de Velilla de San Antonio, donde comienzan los procesos de subsidencia kárstica que inducen la superposición de terrazas en el Jarama (Pérez-González et al., 2013). Estas terrazas, superpuestas en el Jarama, se encuentran también englobadas en el nivel $\mathrm{T}+25-30 \mathrm{~m}$ del Tajo analizado en este estudio (Unidad Pinedo I) y entran dentro del rango cronológico de la Unidad de Arganda II (c. 254-262 ka: MIS 8; Fig. 7), si bien la datación de Pinedo (TOL-8: > $280 \mathrm{ka}$ ) podría entrar dentro del rango cronológico superior de la Unidad Arganda I (c. 332-379 ka: MIS 11-MIS 9; Fig. 7). Más controvertida es la interpretación de la cronología AAR obtenida en la unidad superior Pinedo II (263-189 $\mathrm{ka})$, que indica que el depósito de la $\mathrm{T}+25-30 \mathrm{~m}$ del Tajo pudo continuar hasta el fin del MIS 7 (Fig. 7). Aunque esta muestra presentaba el nivel mínimo de dentina necesario para su datación y su edad ha

Tabla 3.-Tabla de dataciones AAR de molares de equus sp. de Pinedo

\begin{tabular}{lllll}
\hline Código campo & Edad (ka) & Peso (mg) & Ac. Aspártico & D/L Asp. \\
\hline BSL 4764 & - & 40 & - & - \\
BSL 4765 & $226 \pm 37$ & 40,5 & 294 & 0,307 \\
BSL 4766 & - & 14,7 & - & - \\
\hline
\end{tabular}

de tomarse con reservas, la Unidad Arganda II en el Jarama también pudo prolongar su depósito hasta este estadio isotópico, como se desprende de la datación AAR de $174 \pm 20$ ka obtenida para esta unidad en el yacimiento de Valdocarros (Panera et al., 2011).

Los principales yacimientos achelenses de las cuencas de los ríos Duero y Tajo se sitúan en las terrazas medias, a c. +30 m (Santonja \& Pérez-González, 1997,2002 y 2010), lo cual es asimilable a la posición geomorfológica de las terrazas muestreadas en este estudio. Este hecho también se ha constatado para los valles de los grandes ríos de la fachada atlántica del suroeste de Europa (Bridgland et al., 2006) y en concreto para el caso de la Península Ibérica (Santonja \& Pérez-González, 1997, 2002 y 2010; Oosterbeek et al., 2010; Cunha et al., 2012). Estos autores indican que las condiciones medioambientales benignas del MIS 11, período cálido interglaciar que comenzó aproximadamente hace $420 \mathrm{ka}$ (Imbrie et al., 1992), favorecieron el incremento de poblaciones con industria achelense en el suroeste de Europa, siendo antes su presencia poco significativa. No obstante, la preservación de yacimientos en los valles del centro de la Península Ibérica se encuentra favorecido por los procesos de subsidencia kárstica, sobre-sedimentación y consecuente superposición de unidades fluviales. Según los datos geocronológicos existentes (Panera et al., 2011; Pérez-González et al., 2013; Silva et al., 2013), puede decirse que comenzaron durante el MIS 11 (c. $400 \mathrm{ka}$ ), lo que corresponde al depósito de la unidad Arganda I en el Jarama, pero en la zona de Toledo estos procesos comenzarían a partir del inicio del MIS 9 (c. 340 ka), con el depósito de la Unidad Pinedo I en el Tajo (Fig. 7).

En el norte de Francia, destacan las dataciones de los yacimientos achelenses de Cagny-la Garenne (448-443 ka) y Cagny 1'Epinette (318-289 ka) en niveles de terraza situados entre $+40 \mathrm{y}+30 \mathrm{~m}$ en los valles de los ríos Somme y Sena (Tuffreau et al., 2008; Antoine et al., 2010; Limondin-Lozouet et al., 2010). Las edades más recientes del Sena son 


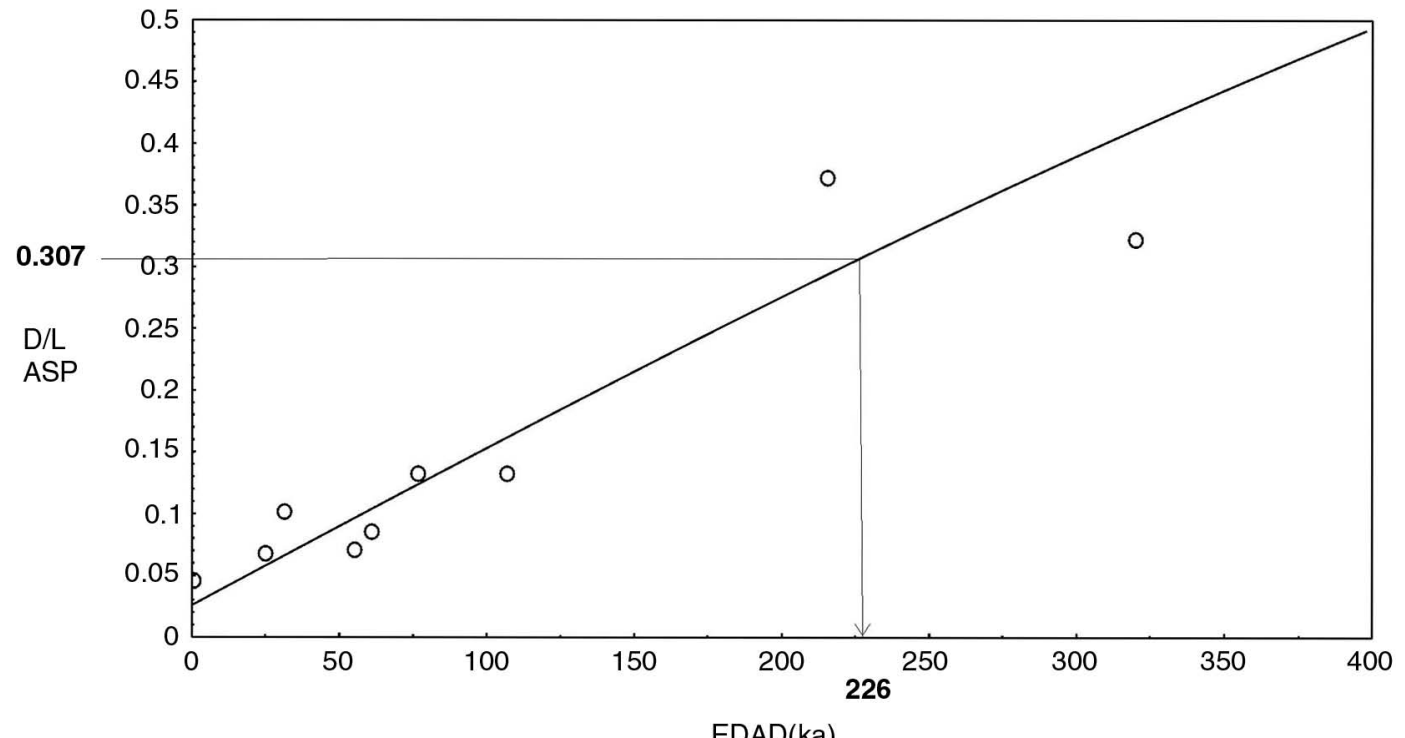

$\operatorname{EDAD}(k a)$

Fig. 6.-Gráfica de datación AAR de un molar de equus sp. de Pinedo (muestra BSL-4765).

similares a las obtenidas en el Tajo para la base de la terraza analizada en este trabajo, situando el desarrollo del nivel a $+30 \mathrm{~m}$ en esta zona de Francia en el tránsito MIS 9-MIS 8. Por otro lado, yacimientos del suroeste de Francia con presencia de industria trifacial, tipo de façonnage característico de los conjuntos líticos de Pinedo y Cien Fanegas, han sido fechados en 284 ka para La Micoque o 239 ka para Barbas C'4 sup (Chevrier, 2006).

En el curso bajo del río Tajo (Portugal), las dataciones OSL disponibles (Cunha et al., 2008, 2012; Martins et al., 2009, 2010) indican que las terrazas de c. $+30-48 \mathrm{~m}$, con edades de c. 260-340 ka, poseen industrias achelenses (Monte do Famaco), mientras que la terraza inmediatamente inferior $(+16-28 \mathrm{~m})$ posee industrias claramente musterienses con fechas comprendidas entre $135 \mathrm{ka}$ (base) y $73 \mathrm{ka}$ (techo), dentro del tránsito Pleistoceno Medio-Superior (MIS 6 - MIS 5). Estas cronologías más recientes son también características de las unidades superiores de las terrazas complejas a $+18-20 \mathrm{~m}$ en los valles del Jarama y Manzanares (Panera et al., 2011; Pérez-González et al., 2013; Silva et al., 2013).

A nivel regional, son comparables a Pinedo y Cien Fanegas, desde el punto de vista tecnológico, los yacimientos achelenses de La Maya II (+34 m; Tormes), El Sartalejo (+26 m; Alagón), San Isidro (+30 m; Manzanares), Puente Pino (depósito sobre la $t+40 \mathrm{~m}$; Tajo) o Tafesa (Terraza Compleja del Manzanares)
(Santonja, 1985; Moloney, 1992; Santonja, 1994; Santonja \& Pérez-González, 1997 y 2002; Rodríguez de Tembleque et al., 2005; Baena et al., 2010a; Silva et al., 2012). Aunque estos yacimientos o bien no disponen de dataciones numéricas o bien no son fiables, los datos faunísticos permiten situarlos en la segunda mitad del Pleistoceno Medio (Sesé et al., 2000). Fuera del ámbito meseteño, son claras las similitudes tecnológicas existentes entre Pinedo y otros conjuntos detectados en depresiones del sistema Subbético (Fuente Camacho -Jiménez-Cobos, 2012-), en el suroeste de Francia o el norte de África.

Fechas comparables para la región central de la Península son las obtenidas por series combinadas de ESR y Uranio de las Unidades Inferiores del yacimiento achelense de Ambrona (Soria) que van desde los $286 \mathrm{ka}$ a los $366 \mathrm{ka}$ (Falguères et al., 2006). A su vez, las fechas obtenidas por el mismo método por luminiscencia pIR-IR y quartz TT-OSL en la Trinchera Galería de Atapuerca, donde se conservan conjuntos achelenses (Ollé et al., 2013) son $313 \pm 14$ ka para la base de la Unidad GIIa y c. 240 ka para la Unidad GIIb-GIV (Demuro et al., 2014), siendo cronologías similares a las de los depósitos de Pinedo y Cien Fanegas.

Debe advertirse que en el entorno que estudiamos la proximidad cronológica de procesos tecnológicos diferentes entre sí durante el último tercio del Pleistoceno Medio es una constante, existiendo una 


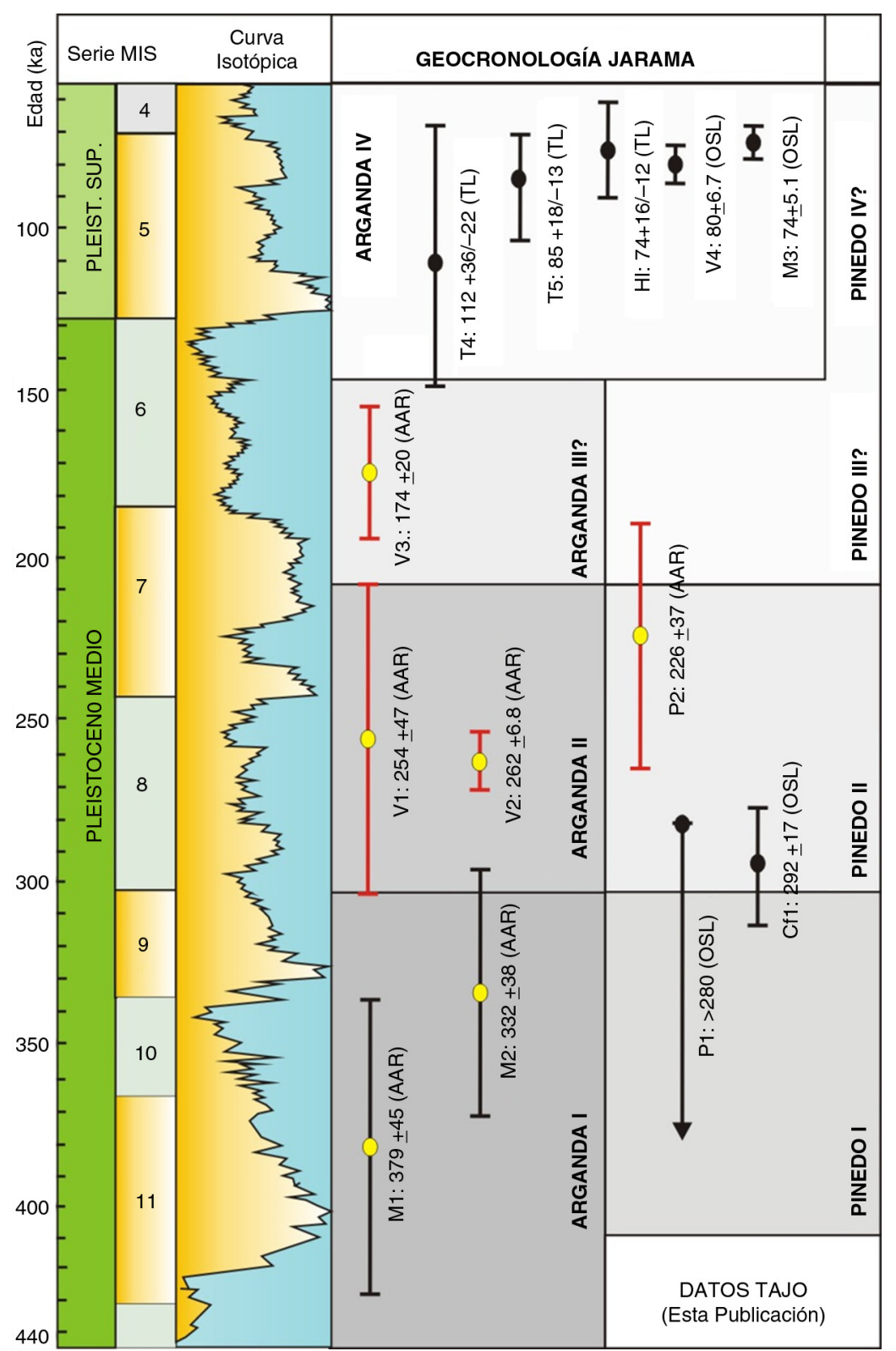

Fig. 7.-Posición estratigráfica de los yacimientos de Pinedo y Cien Fanegas en relación con la geocronología del valle del Jarama y estadios isotópicos del Pleistoceno Inferior y Medio según Imbrie et al. (1992).

gran variabilidad sobre todo entre complejos líticos del Paleolítico Medio antiguo y achelenses.

\section{Discusión y conclusiones}

Las edades OSL (pIRIR) presentadas en este estudio indican que los depósitos fluviales correspondientes a las terrazas de $+25-30$ m en Pinedo y Cien Fanegas se depositaron en un lapso de tiempo comprendido entre el MIS 9 ( $>280 \mathrm{ka})$ y el MIS 8 (c. $292 \mathrm{ka}$ ), aunque la edad AAR obtenida en Pinedo (c. $226 \mathrm{ka}$ ) podría indicar que su depósito continuó hasta el MIS 7. Teniendo en cuenta los datos geocronológicos regionales, la terraza analizada y los yacimientos achelenses y faunísticos que contienen sus niveles basales, entran dentro del rango cronológico de la Unidad Arganda II $(\mathrm{T}+25 \mathrm{~m})$ documentada en el valle del Jarama (Fig. 7). 
La geocronología de esta unidad indica que su desarrollo continuó hasta poco después del fin del MIS 7 (174 20 ka; Panera et al., 2011), como también puede ocurrir en el valle del Tajo teniendo en cuenta la edad AAR del yacimiento de Pinedo que se aporta en este trabajo. No obstante, la incertidumbre de la fecha obtenida en Pinedo ( $>280 \mathrm{ka})$, reforzada por la edad de Cien Fanegas (292 $\pm 17 \mathrm{ka}$ ) obtenidas en sus unidades basales (Pinedo I), podría indicar que el depósito de esta unidad basal podría ser algo anterior, correlacionándose con la Unidad Arganda I del Jarama (Fig. 7), con la que además comparte registros faunísticos (Elephas antiquus) y líticos (achelense) similares, y ligeramente diferentes a los que presenta la terraza inmediatamente anterior en la zona $(+40 \mathrm{~m})$. La presencia de Mammuthus trogontherii y las características de los micromamíferos del nivel de $+40 \mathrm{~m}$ en la zona indicarían una edad del Pleistoceno Medio anterior a los yacimientos de Arganda (Sesé et al., 2000), existiendo una posible equivalencia cronológica entre los yacimientos del Jarama y Pinedo que se propone en este estudio en función de la geocronología resultante (Fig. 7).

Por otro lado, la datación de los depósitos aluviales (Pinedo IV; TOL-7) que erosionan la formación Pinedo arroja una edad de $11,8 \pm 1,3$ ka por quartz-OSL y 11,0+0,9 ka por pIRIR (Tabla 2), que se sitúa en el Alleröd, la fase menos fría del Tardiglaciar. En cualquier caso, la presencia de formaciones aluviales más recientes erosionando o cubriendo las superficies de las terrazas es común en los valles del Tajo, Jarama y Manzanares, pudiendo comprender edades entre los c.70-60 y $11 \mathrm{ka}$ (MIS 4-MIS 2) para niveles de terraza inferiores a los $+40 \mathrm{~m}$ (Panera et al., 2011; Silva et al., 2013), incluyendo los niveles de terraza más bajos (Wolf et al., 2013). En su mayor parte responden a depósitos aluviales laterales que provienen de la degradación de niveles de terrazas superiores o del sustrato mioceno adyacente. Todos ellos se producen durante el último ciclo glaciar a la par que se desarrollan los niveles de terraza inferiores a +12 (c. $40 \mathrm{ka}),+8-9$ (c. $25-30 \mathrm{ka}) \mathrm{y}+5 \mathrm{~m}$ (c. $18-21 \mathrm{ka})$ durante los eventos Heinrich (Pérez-González et al., 2008; Silva et al., 2013). En el caso de los depósitos relacionados con la terraza analizada en este estudio, muestran un claro retoque eólico (ventifactos, Cien Fanegas) evidenciando la prevalencia de condiciones áridas y frías durante todo este periodo.
Son numerosas las referencias a yacimientos con tecnología achelense en terrazas fluviales en el suroeste europeo contextualizados en el MIS 9 (340-300 ka) y durante el comienzo del MIS 8 (300-245 ka) en niveles de terraza de altimetría similar $(+25-30 \mathrm{~m})$ a los analizados en el valle medio del Tajo. Este hecho puede deberse en gran medida a la propia dinámica sedimentaria que favorece la formación de los yacimientos ligada a fenómenos de agradación fluvial durante el final de periodos fríos (ej. MIS 8 y MIS 10) y la parte inicial de los periodos cálidos que los siguen (ej. MIS 9 y MIS 7); esto es, con las denominadas "terminaciones", tal como sugieren Blain et al. (2012) para el yacimiento de Valdocarros en el Jarama. Durante los periodos cálidos se estabilizarían las llanuras de inundación, permitiendo la expansión de bosques mediterráneos en el interior de los valles y el desarrollo de suelos con horizontes argilíticos (Bt) bien desarrollados (ej. Cien Fanegas), como sugieren los datos paleoclimáticos y edáficos en los valles del Jarama y del Tajo (Blain et al., 2012; Roquero et al., 2015). Los fenómenos de encajamiento fluvial que permiten la formación de terrazas escalonadas se desarrollarían fundamentalmente durante el inicio de los periodos fríos acorde al progresivo descenso del nivel de base asociado (bajadas del nivel del mar). En cualquier caso, las particulares condiciones geológicas que registran los valles del Tajo y Jarama, sujetos a un continuo proceso de subsidencia kárstica de las evaporitas, desde al menos el inicio del MIS 9, hacen que los procesos de agradación fluvial que favorecen la preservación de yacimientos prevalezcan hasta al menos el final del MIS 5.

En el valle del Tajo el proceso de sobresedimentación y agradación fluvial está condicionado por los procesos de subsidencia que se registran aguas arriba de Toledo, probablemente mantenidos y amplificados por la existencia del "cuello de botella" que supone el Meandro encajado de Toledo. Éste, desarrollado sobre los materiales cristalinos de los Montes de Toledo, funcionaría como un nivel de base interno "inactivo" para toda la cuenca fluvial del Tajo situada aguas arriba. En la actualidad el desnivel del cauce del Tajo entre la entrada y la salida del Meandro encajado es de 10-12 m, por lo tanto hay que tomar mucha precaución en la correlación 
altimétrica de los niveles de terraza situados aguas arriba y aguas abajo del mismo. No obstante este proceso no afecta a la correlación de los yacimientos y terrazas analizados en este estudio, todos ellos situados aguas arriba de Toledo y sujetos a similar dinámica de encajamiento y sedimentación fluvial.

Finalmente, conviene resaltar que la metodología post-IR IRSL (Buylaert et al., 2012) empleada en este estudio ha permitido ofrecer las edades OSL más antiguas hasta la fecha (c. $292 \mathrm{ka}$ ) para sedimentos fluviales en la cuenca del Tajo, si bien las dataciones más antiguas son aquellas obtenidas mediante AAR en el Jarama (c. $379 \mathrm{ka}$ ) para la unidad Arganda I (Panera et al., 2011). Como se ha comentado a lo largo del trabajo, esta metodología también se ha ensayado para niveles de terraza más altos a $+40,+50 \mathrm{y}+60 \mathrm{~m}$, situados aguas abajo de Toledo, cuyos resultados se encuentran actualmente en proceso de análisis e interpretación. Dichos resultados permitirán obtener un primer marco geocronológico para el Pleistoceno Medio y Superior del valle del Tajo en la zona central de la Península Ibérica, tomando las debidas precauciones impuestas por la presencia del nivel de base interno que supone el Meandro encajado de Toledo.

\section{AGRADECIMIENTOS}

El presente trabajo ha sido financiado por la Junta de Comunidades de Castilla-La Mancha mediante los proyectos de investigación "Una aproximación al conocimiento del Pleistoceno en la cuenca media del río Tajo (Toledo) a través de las colecciones arqueológicas y paleontológicas de D. Máximo Martín Aguado" y "Estudio cronoestratigráfico de las terrazas de Pleistoceno Medio y Superior para la reconstrucción paleoambiental del valle del río Tajo (Toledo)". La investigación ha sido también financiada parcialmente por AUDEMA S.A., IMAR-CMA (Univ. Coimbra, Portugal), Aarhus Univ. y Risøe DTU (Denmark) y por el proyecto de investigación español CGL2012-37281-C02.01 (USAL). Este trabajo se incluye en el proyecto HAR2010-2015 "Algo más que bifaces: hacia la definición técnica y tecnológica de los conjuntos líticos del Pleistoceno de la región de Madrid" del Ministerio de Economía y Competitividad. Agradecemos a Inmaculada Rus haber podido revisar el material lítico de Cien Fanegas. Este trabajo es una contribución del Grupo de Cuaternario Madrileño (GQM) de AEQUA. Agradecemos a los revisores anónimos y a David Uribelarrea los comentarios aportados.

\section{Referencias}

Aguirre, E. (1965). Los elefantes de las terrazas medias de Toledo y la edad de estos depósitos. Coloquios de Paleontología, 4: 7-8.
Alférez, F. (1977). Estudio del sistema de terrazas del Tajo al W de Toledo. Estudios Geológicos, 33 (3): 223-250.

Alférez, F. (1978). Presencia de Equus stenonis Cocchi en el Pleistoceno inferior de la cuenca del Tajo. Estudios Geológicos, 34: 553-558.

Antoine, P.; Auguste, P.; Bahain, J.J.; Chaussé, C.; Falguères, C.; Ghaleb, B.; Limondin-Lozouet, N.; Locht, J.L. \& Voinchet, P. (2010). Chronostratigraphy and palaeoenvironments of Acheulean occupations in northern France (Somme, Seine and Yonne valleys). Quaternary International, 223-224: 456-461. http:// dx.doi.org/10.1016/j.quaint.2009.07.014.

Baena, J. \& Baquedano, I. (2004). Avance de los trabajos arqueológicos realizados en el yacimiento paleolítico de TAFESA, antiguo TRANSFESA (Villaverde-Madrid): principales rasgos tecnológicos del conjunto. In: Miscelánea en Homenaje a Emiliano Aguirre, vol. 4. Museo Arqueológico Regional, Madrid, 30-47.

Baena, J.; Baquedano, I. \& Carrión, E. (2010a). La industria lítica del yacimiento paleolítico de TAFESA (Madrid). In: Las Huellas de nuestro pasado. Estudio del yacimiento del Pleistoceno madrileño de Tafesa (Antigua Transfesa) (Baena Preysler, J. \& Baquedano Beltrán, I. Coords.), Zona Arqueológica, 14. Museo Arqueológico Regional, Madrid, 37-134.

Baena, J.; Rus, I.; Cuartero, F.; Martín, D.; Rubio, D. \& Roca, M. (2010b). Estudio tecnológico de la industria lítica del yacimiento de Las Cien Fanegas (Madrid) en el contexto Achelense de la Meseta. Cuaternario y Arqueología. Homenaje a Francisco Giles Pacheco. Diputación Provincial de Cádiz, Cádiz, 71-83.

Blain, H.A.; Panera, J.; Uribelarrea, D.; Rubio-Jara, S. \& Pérez-González, A. (2012). Characterization of a rapid climate shift at the MIS 8/7 transition in central Spain (Valdocarros II, Madrid) by means of the herpetological assemblages. Quaternary Science Reviews, 47: 73-81. http://dx.doi.org/10.1016/j. quascirev.2012.05.021.

Bridgland, D.R.; Antoine, P.; Limondin-Lozouet, N.; Santisteban, J.J.; Westaway, R. \& White, M.J. (2006). The Palaeolithic occupation of Europe as revealed by evidence from the rivers: data from IGCP 449. Journal of Quaternary Science, 21: 437-455. http:// dx.doi.org/10.1002/jqs.1042.

Buylaert, J.P.; Murray, A.S.; Thomsen, K.J. \& Jain, M. (2009). Testing the potential of an elevated temperature IRSL signal from K-feldspar. Radiation Measurements, 44 (5-6): 560-565. http://dx.doi. org/10.1016/j.radmeas.2009.02.007.

Buylaert, J.P.; Jain, M.; Murray, A.; Thomsen, K.J.; Thiel, C. \& Sohbati, R. (2012). A robust feldspar luminescence dating method for Middle and Late Pleistocene sediments. Boreas, 41: 435-451. http:// dx.doi.org/10.1111/j.1502-3885.2012.00248.x.

Chévrier, B. (2006). De l'Acheuléen méridional au technocomplexe trifacial: la face cachée des industries 
du Bergeracois. Gallia Préhistoire, 48: 207-252. http:// dx.doi.org/10.3406/galip.2006.2451.

Cunha, P.P.; Martins, A.A.; Huot, S.; Murray, A. \& Raposo, L. (2008). Dating the Tejo River lower terraces in the Ródão area (Portugal) to assess the role of tectonics and uplift. Geomorphology, 102: 43-54. http://dx.doi. org/10.1016/j.geomorph.2007.05.019.

Cunha, P.P.; Almeida, N.; Aubry, T.; Martins, A.A.; Murray, A.S.; Buylaert, J.-P.; Sohbati, R.; Raposo, L. \& Rocha, L. (2012). Records of human occupation from Pleistocene river terrace and aeolian sediments in the Arneiro depression (Lower Tejo River, central eastern Portugal). Geomorphology, 165-166: 78-90. http://dx.doi.org/10.1016/j.geomorph.2012. 02.017 .

De Combes, L. (1906). Prehistoria del distrito de Talavera de la Reina. Universidad Central, 50 pp.

Del Pan, I. (1925). Restos fósiles de Proboscídeos existentes en el Gabinete de Historia Natural del Instituto de Toledo. Boletín de la Real Sociedad Española de Historia Natural, 25: 343-352.

Demuro, M.; Arnold, L.J.; Parés, J.M.; Pérez-González, A.; Ortega, A.I.; Arsuaga, J.L.; Bermúdez de Castro, J.M. \& Carbonell, E. (2014). New luminescence ages for the Galería Complex archaeological site: resolving chronological uncertainties on the acheulean record of the Sierra de Atapuerca, Northern Spain. PLOS ONE, 9 (10): 1-24. http://dx.doi.org/10.1371/journal. pone. 0110169 .

Falguères, C.; Bahain, J.J.; Pérez-González, A.; Mercier, N.; Santonja, M. \& Dolo, J.M. (2006). The Lower Acheulian site of Ambrona, Soria (Spain): ages derived from a combined ESR/U-series model. Journal of Archaeological Science, 33: 149-157. http://dx.doi.org/10.1016/j.jas.2005.07.006.

Hernández-Pacheco, E. (1930). El Meandro Encajado del Tajo en torno a Toledo. Boletín de la Real Sociedad Española de Historia Natural, 30: 116-119.

Herrero Matías, M. (1988). Mapa geomorfológico de Toledo-Sonseca a escala 1:50.000. IGN-UCM, Madrid, $41 \mathrm{pp}$.

IGME (In press). Hoja geológica 629 (Toledo). Cartografía Geológica de España, escala 1:50.000, Madrid.

Imbrie, J.; Boyle, E.A.; Clemens, S.C.; Duffy, A.; Howard, W.R.; Kukla, G.; Kutzbach, J.; Martinson, D.G.; Mc Intyre, A.; Mix, A.C.; Molfino, B.; Morley, J.J.; Peterson, L.C.; Pisias, N.G.; Prell, W.L.; Raymo, M.E.; Shackleton, N.J. \& Toggweiler, J.R. (1992). On the structure and origin of mayor glaciation cycles (1): Linear responses to Milankovitch forcing. Paleoceanography, 7 (6): 701-738. http:// dx.doi.org/10.1029/92PA02253.

Jiménez-Cobos, F. (2012). Tecnología y análisis diacrítico. Aproximación al yacimiento del Cortijo del Calvillo (Fuente Camacho, Loja) a través de la colección del Museo de la Alcazaba de Loja (Granada). Arqueología y Territorio, 9: 19-32.
Limondin-Lozouet, N.; Nicoud, E.; Antoine, P.; Auguste, P.; Bahain, J.J.; Dabkowski, J.; Dupéron, J.; Dupéron, M.; Falguères, C.; Ghaleb, B.; Jolly-Saad, M.C. \& Mercier, N. (2010). Oldest evidence of Acheulean occupation in the Upper Seine valley (France) from an MIS 11 tufa at La Celle. Quaternary International, 223-224: 299-311. http://dx.doi.org/10.1016/j.quaint. 2009.10.013.

Martín Aguado, M. (1962). Recientes hallazgos prehistóricos en la gravera de Toledo. Estudios Geológicos, 18 (3-4): 139-154.

Martín Aguado, M. (1963). El yacimiento prehistórico de Pinedo (Toledo) y su industria triédrica. Diputación de Toledo, Toledo, 156 pp.

Martín Aguado, M. (1990). El origen del Torno del Tajo en Toledo y sus implicaciones geomorfológicas y prehistóricas. Toletum, 24: 39-110.

Martins, A.A.; Cunha, P.P.; Huot, S.; Murray, A.S. \& Buylaert, J.P. (2009). Geomorphological correlation of the tectonically displaced Tejo River terraces (Gavião-Chamusca area, central Portugal) supported by luminescence dating. Quaternary International, 199: 75-91. http://dx.doi.org/10.1016/j.quaint.2009. 01.009 .

Martins, A.A.; Cunha, P.P.; Rosina, P.; Oosterbeck, L.; Cura, S.; Grimaldi, S.; Gomes, J.; Buylaert, J.-P.; Murray, A.S. \& Matos, J. (2010). Geoarchaeology of Pleistocene open-air sites in the Vila Nova da Barquinha - Santa Cita area (Lower Tejo River basin, central Portugal). Proceedings of the Geologists' Association, 121 (2): 128-140. http://dx.doi. org/10.1016/j.pgeola.2010.01.005.

Moloney, N. (1992). Lithic production and raw material exploitation at the Middle Pleistocene site of El Sartalejo, Spain. Papers from the Institute of Archaeology, 3: 11-22. http://dx.doi.org/10.5334/pia.35.

Moreno, D.; Falguères, C.; Pérez-González, A.; Duval, M.; Voinchet, P.; Benito-Calvo, A.; Ortega, A.I.; Bahain, J.J.; Sala, R.; Carbonell, E.; Bermúdez de Castro, J.M. \& Arsuaga, J.L. (2012). ESR chronology of alluvial deposits in the Arlanzón valley (Atapuerca, Spain): contemporaneity with Atapuerca Gran Dolina site. Quaternary Geochronology, 10: 418-423. http://dx.doi.org/10.1016/j. quageo.2012.04.018.

Murray, A.S. \& Olley, J.M. (2002). Precision and accuracy in the optically stimulated luminescence dating of sedimentary quartz: a status review. Geochronometria, 21: 1-16.

Ollé, A.; Mosquera, M.; Rodríguez, X.P.; Lombera-Hermida, A. de; García-Antón, $M^{a}$ D.; García-Medrano, P.; Peña, L.; Menéndez, L.; Navazo, M.; Terradillos, M.; Bargalló, A.; Márquez, B.; Sala, R. \& Carbonell, E. (2013). The Early and Middle Pleistocene technological record from Sierra de Atapuerca (Burgos, Spain). Quaternary International, 295: 138-167. http://dx.doi. org/10.1016/j.quaint.2011.11.009. 
Oosterbeck, L.; Grimaldi, S.; Rosina, P.; Cura, S.; Cunha, P.P. \& Martins, A.A. (2010). The earliest Pleistocene archaeological sites in western Iberia: present evidences and research prospects. Quaternary International, 223-224: 399-407. http://dx.doi. org/10.1016/j.quaint.2010.01.024.

Panera, J.; Rubio-Jara, S.; Pérez-González, A. \& Uribelarrea, D. (2008). Estudo xeoarqueolóxico das terrazas complexas dos vales Manzanares e Jarama (Madrid). In: Estudos sobre Paleolítico Homenaxe a Xosé María Álvarez Blázquez (Méndez Quintas, E., Coord.), Instituto de Estudos Miñoranos, Vigo, 223-242.

Panera, J.; Torres, T.; Pérez-González, A.; Ortiz, J.E.; Rubio-Jara, S. \& Uribelarrea Del Val, D. (2011). Geocronología de la Terraza Compleja de Arganda en el valle del río Jarama (Madrid, España). Estudios Geológicos, 67 (2): 495-504. http://dx.doi.org/ 10.3989/egeol.40550.204.

Pérez de Barradas, J. (1920). Algunos datos sobre el Cuaternario de las inmediaciones de Toledo. Boletín de la Real Academia de Bellas Artes y Ciencias Históricas de Toledo, 8-9: 229-231.

Pérez-González, A. (1982). Neógeno y Cuaternario de la Llanura manchega y sus relaciones con la Cuenca del Tajo. Tesis doctoral, Universidad Complutense de Madrid, 787 pp.

Pérez-González, A. (1994). Depresión del Tajo. In: Geomorfología de España (Gutiérrez Elorza, M. Ed.). Editorial Rueda, Madrid, 389-436.

Pérez-González, A.; Gallardo-Millán, J.L.; Uribelarrea del Val, D.; Panera, J. \& Rubio-Jara, S. (2013). La inversión Matuyama-Brunhes en la secuencia de terrazas del río Jarama entre Velilla de San Antonio y Altos de la Mejorada, al SE de Madrid (España). Estudios Geológicos, 69 (1): 35-46. http://dx.doi. org/10.3989/egeol.40862.173.

Pinilla, L.; Pérez-González, A.; Sopeña, A. \& Parés, A. (1995). Fenómenos de hundimientos sinsedimentarios en los depósitos cuaternarios del río Tajo en la Cuenca de Madrid (Almoguera-Fuentidueña de Tajo). Monografías del Centro de Ciencias Medioambientales, 3: 125-139.

Querol, M.A. \& Santonja, M. (1979). El yacimiento achelense de Pinedo (Toledo). Excavaciones Arqueológicas en España, 106. Ministerio de Cultura, Madrid, $181 \mathrm{pp}$.

Rodríguez de Tembleque, J.M. (2008). Primeras ocupaciones humanas en la Meseta española: Estudio geoarqueológico de depósitos fluviales en la cuenca media del Tajo. Tesis doctoral, Universidad Autónoma de Madrid. Servicio de Publicaciones UAM, 843 pp. http://hdl.handle.net/10486/2395.

Rodríguez de Tembleque, J.M.; Santonja, M. \& PérezGonzález, A. (2005). Puente Pino: un yacimiento achelense en Alcolea de Tajo (Toledo, España). In: Geoarqueología y Patrimonio en la Península Ibérica y el entorno mediterráneo (Santonja, M., PérezGonzález, A. \& Machado, Mª., Eds.), ADEMA, Soria, 283-295.

Rodríguez de Tembleque, J.M.; Pérez-González, A. \& Santonja, M. (2010). Indicios de presencia humana en la Meseta Ibérica durante la formación de la Terraza +75-80 m del Río Tajo. Cuaternario y Arqueología. Homenaje a Francisco Giles Pacheco. Diputación Provincial de Cádiz, Cádiz, 45-54.

Roquero, E.; Goy, J.L. \& Zazo, C. (1999). Fenómenos de convergencia genética en suelos de terrazas fluviales: Valle del Río Tajo, Madrid-Toledo (España). Revista de la Sociedad Geológica de España, 12 (3-4): 329-338.

Roquero, E.; Silva, P.G.; Goy, J.L.; Zazo, C. \& Massana, J. (2015). Soil evolution indices in fluvial terrace chronosequences of Central Spain (Tagus and Duero fluvial basins). Quaternary International, en prensa. http://dx.doi.org/10.1016/j.quaint.2014.11.036.

Ruiz Zapata, M.B.; Rodríguez de Tembleque, J.M.; Pérez-González, A.; Gil García, M.J. \& Santonja, M. (2009). Paleovegetación en el yacimiento achelense de Puente Pino y su entorno (Toledo, España). Cuaternario y Geomorfología, 23 (1-2): 113-126.

Rus, I.; Roquero, E.; Mazo, A. \& Enamorado, J. (1993). Terrazas del Pleistoceno Medio en la Cuenca del Tajo y fauna e industria asociada. 3 Reuniao do Cuaternario Iberico. AEQUA, Coimbra, 86.

Santonja, M. (1981). El Paleolítico Inferior de la Meseta Central española. Tesis doctoral, Universidad Complutense de Madrid, 3 vol.

Santonja, M. (1985). El yacimiento achelense de El Sartalejo (Valle del Alagón, Cáceres). Estudio preliminar. Series de Arqueología Extremeña, 2. Universidad de Extremadura, Cáceres, 109 pp.

Santonja, M. (1992). La adaptación al medio en el Paleolítico inferior de la Península Ibérica. Elementos para una reflexión. In: Elefantes, Ciervos y Ovicaprinos. Economía y aprovechamiento del medio en la Prehistoria de España y Portugal (Moure Romanillo, A., Ed.), Universidad de Cantabria, Santander, 37-76.

Santonja, M. (1994). Los últimos diez años en la investigación del Paleolítico inferior de la cuenca del Duero. Veleia, 8-9: 7-41.

Santonja, M. \& Querol, A. (1982). Industrias del Paleolítico Inferior arcaico en la Meseta española. In: En homenaje a Conchita Fernández Chicarro. Ministerio de Cultura, Madrid, 17-31.

Santonja, M. \& Pérez-González, A. (1997). Los yacimientos achelenses en terrazas fluviales de la Meseta Central española. In: Cuaternario Ibérico (Rodríguez Vidal, J., Ed.). AEQUA, Huelva, 224-234.

Santonja, M. \& Pérez-González, A. (2002). El Paleolítico inferior en el interior de la Península ibérica. Un punto de vista desde la geoarqueología. Zephyrus, 53-54: 27-77. 
Santonja, M. \& Villa, P. (2006). The Acheulean in Southwestern Europe. In: Axe Age. Acheulian Tool-making from Quarry to Discard (Goren-Inbar, N. \& Sharon, G., Eds.). Equinox Publishing, London, 429-478.

Santonja, M. \& Pérez-González, A. (2010). Mid-Pleistocene Acheulean industrial complex in the Iberian Peninsula. Quaternary International, 223-224: 154-161. http:// dx.doi.org/10.1016/j.quaint.2010.02.010.

Sesé, C.; Soto, E. \& Pérez-González, A. (2000). Mamíferos de las terrazas del valle del Tajo: primeros datos de micromamíferos del Pleistoceno de Toledo (España central). Geogaceta, 28: 137-140.

Silva, P.G.; López-Recio, M.; Cuartero, F.; Baena, J.; Tapias, F.; Manzano, I.; Martín, D.; Morín, J. \& Roquero, E. (2012). Contexto geomorfológico y principales rasgos tecnológicos de nuevos yacimientos del Pleistoceno Medio y Superior en el Valle Inferior del Manzanares (Madrid, España). Estudios Geológicos, 68 (1): 57-89. http://dx.doi.org/10.3989/egeol.40338.134.

Silva, P.G.; López-Recio, M.; Tapias, F.; Roquero, E.; Morín, J.; Rus, I.; Carrasco-García, P.; Giner-Robles, J.L.; Rodríguez-Pascua, M.A. \& Pérez-López, R.
(2013). Stratigraphy of the Arriaga Palaeolithic sites. Implications for the geomorphological evolution recorded by thickened fluvial sequences within the Manzanares River valley (Madrid Neogene Basin, Central Spain). Geomorphology, 196: 138-161. http:// dx.doi.org/10.1016/j.geomorph.2012.10.019.

Torres, T.; Ortiz, J.E.; Fernández, E.; Arroyo-Pardo, E.; Grün, R. \& Pérez-González, A. (2014). Aspartic acid racemization as a dating tool for dentine: a reality. Quaternary Geochronology, 22: 43-56. http://dx.doi. org/10.1016/j.quageo.2014.02.004.

Tuffreau, A.; Lamotte, A. \& Goval, E. (2008). Les industries acheuléennes de la France septentrionale. L'Anthropologie, 112: 104-139. http://dx.doi.org/ 10.1016/j.anthro.2008.01.003.

Vaudour, J. (1979). La Región de Madrid, altérations, sols et paléosols. Editorial Ophrys, Paris, 390 pp.

Wolf, D.; Seim, A.; Díaz del Olmo, F. \& Faust, D. (2013). Late Quaternary fluvial dynamics of the Jarama River in central Spain. Quaternary International, 302: 20-41. http://dx.doi.org/10.1016/j. quaint.2013.02.012. 